Diversitates International Journal (ISSN: 1984-5073)

Vol. 12, N.2, Julho/Dezembro (2020), p. 131 - 162

Recebido em: 27/06/2020

Aceito em: 30/06/2020

\title{
Protocolos em tempos de pandemia por COVID-19 com critérios para alocação de recursos escassos: podem considerá-los
} (in)justos?

Protocols in times of pandemic by COVID-19 with criteria for allocating scarce resources: can you consider them (un) fair?

Alexandre Costa ${ }^{a}$

Cristiane Maria Amorim Costab Fabiana Cunha Leão Pompermayer ${ }^{\mathrm{c}}$

Resumo: Este artigo tem por objetivo discutir sobre os protocolos para alocação de recursos escassos durante a pandemia por COVID-19. Inicialmente, é realizado um panorama sobre a pandemia e seus desdobramentos, sendo apresentado os protocolos de alocação de recursos escassos propostos, a partir de uma breve análise dos fundamentos éticos e clínicos/técnicos que sustentam tais protocolos. Nesta visita, são levantadas algumas consequentes fragilidades e desigualdades de acesso da população em geral ao tratamento. A seguir, é deflagrado apontamentos críticos sobre a construção dos protocolos e dos participantes dos comitês deliberativos, responsáveis por alargar as desigualdades e injustiças à população brasileira. Por fim, propomos, não uma conclusão, mas uma abertura ao debate, subsidiada por questões de justiça distributiva e uma compreensão ampliada sobre as entranhas que envolvem as escolhas de fazer viver e deixar morrer.

Palavras-chaves: Pandemia / Planejamento em Saúde / Ética / Direito à Saúde

Abstract: This article aims to discuss the protocols for allocating scarce resources during the COVID-19 pandemic. Initially, an overview of the pandemic and its consequences is made, and the proposed scarce resource allocation protocols are presented, based on a brief analysis of the ethical and clinical / technical foundations that support such protocols. In this visit, some consequent weaknesses and inequalities in access of the general population to treatment are raised. Next, critical notes about the construction of the protocols and the participants of the deliberative committees, responsible for extending inequalities and injustices to the Brazilian population, are triggered. Finally, we propose, not a conclusion, but an openness to debate, subsidized by issues of distributive justice and a broader understanding of the guts that involve the choices to live and let die.

Keywords: Pandemic / Health Planning / Ethic / Right to Health

\footnotetext{
a Professor do PPGBIOS e do PPGF, ambos UFRJ.

${ }^{\text {b }}$ Professora Adjunta da Faculdade de Enfermagem UERJ, Professora Permanente do Programa de

Bioétca, Ética Aplicada e Saúde Coletiva

${ }^{\mathrm{C}}$ Fonoaudióloga, Neurocientista e Doutoranda em Bioética, Ética Aplicada e Saúde Coletiva (PPGBIOS)
}

Copyright (c) 2020 Costa et al. Este é um artigo em acesso aberto distribuído nos termos da Licença Creative Commons. Atribuição que permite o uso irrestrito, a distribuição e reprodução em qualquer meio desde que o artigo original seja devidamente citado.

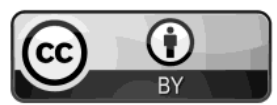




\section{Contextualizando a pandemia e seus desdobramentos}

A princípio, nos séculos anteriores, acreditava-se que 2020 seria já um ano de muitas inovações na história da humanidade. O despertar do século XXI traria à cena um mundo repleto de automação. Um completo domínio da ciência e da tecnologia sobre a vida biológica. Entretanto, já vinham acontecendo pandemias como a Influenza e Ebola, que também traziam preocupações com a saúde global da população e com o risco de futuras pandemias. Sendo imprescindível haver planejamento para um futuro acontecimento, como refere Ross et al (2015) e Ferreira et al (2014/2020), que abordaram sobre a importância da organização do acesso à saúde no combate às futuras pandemias. A preocupação se consolidou, mas sem o devido planejamento a partir da construção de regulamentos internacionais de saúde e fortalecimento dos sistemas de saúde.

A Organização Mundial da Saúde (OMS), declarou em 30 de janeiro deste ano, emergência de saúde pública de interesse internacional, por conta de um vírus encontrado em Wuhan, capital de Hubei, província na região central da China, em dezembro de 2019, denominado COVID-19¹ (SOHRAB et al. 2020). Este vírus possui alta capacidade de transmissibilidade e rápida propagação globalizada. Em 11 de março, a OMS reconheceu o surto como pandemia e anunciou que 115 países possuíam casos declarados da infecção com 118.319 casos confirmados e 4.292 mortes $^{2}$. Em Junho de 2020, a OMS divulga 7.941.791 de casos confirmados e 434.796 mortes $^{3}$ no mundo.

Frente à imperiosa necessidade de implementação de ações de enfrentamento da pandemia por COVID-19, o mundo se deparou com importantes questões éticas e bioéticas, relacionadas aos cuidados de saúde para pacientes com acometimentos 
graves e que necessitam de recursos médico-hospitalares. A cena dos hospitais ao redor do mundo é de sobrecarga: profissionais da saúde, leitos e respiradores, cada vez mais distantes da capacidade de atender a todos os doentes com a dignidade necessária, passa a assolar o mundo. Surge a catástrofe das escolhas que implicam em fazer viver e deixar morrer.

O Brasil teve seu primeiro caso confirmado em 26 de fevereiro, com a primeira morte por COVID-19 em 16 de março, em São Paulo4. Em Junho de 2020 já foram confirmados 923.189 e 45.241 mortes $^{5}$. Como uma doença de alta transmissibilidade, a COVID-19 possui um risco de complicação alto em que uma parcela significante, cerca de $20 \%$ da população infectada irão precisar de internação ao longo de 12 meses, sendo que $5 \%$ irão demandar cuidados intensivos ${ }^{6}$ (Conselho Nacional de Saúde, 2020).

Entretanto, o sistema de saúde, que tem como princípios absolutos a universalidade e a equidade ${ }^{7}$, está sucateado, não atendendo às reais necessidades da população em geral. Está associado à carência de locais para atendimento o falho sistema de regulação do Estado, que compromete a regulação para atendimento e para a ocupação de leitos. É notório a escassez de profissionais dentro dos hospitais públicos, e a precarização do trabalho com contratos sem nenhum direito trabalhista para minimizar os efeitos desta situação. E, por fim, os recursos materiais são escassos com carência de insumos básicos, tecnologias necessárias ao tratamento e cuidado do usuário, entre outros.

A escassez de recursos passou a ser uma realidade imposta a quase todos os países do mundo e agravada no Brasil, acarretando um acréscimo de estresse cotidiano aos profissionais de saúde. A situação é difícil e preocupante, o sistema de 
saúde e os profissionais trabalham em exaustão. Comparado com países da Europa, o Brasil lida com essa situação rotineiramente, sem pandemia. O que torna tudo muito pior em meio a esta catástrofe.

Segundo dados do Instituto de Estudos Socioeconômicos ${ }^{8}$, divulgados em seu Balanço do Orçamento Geral da União 2019 "O Brasil com baixa imunidade", o orçamento destinado à saúde desde 2014 vinha em uma linha decrescente, e em 2019, estabilizou em patamares semelhantes a 2014. Porém houve um aumento populacional no Brasil de 7 milhões, aumentando, por consequência, as demandas em saúde. Conforme aponta o documento:

O desfinanciamento da saúde limitou a capacidade de uma resposta rápida e eficiente à pandemia da Covid-19, prejudicando principalmente as populações mais vulneráveis, que dependem exclusivamente do SUS. Se este fosse corretamente financiado, talvez tivéssemos uma quantidade adequada de leitos de UTI, respiradores, equipamentos de proteção individual e testes para lidar com a situação, além de mais profissionais de saúde, em especial os não médicos, como enfermeiros, fisioterapeutas e agentes comunitários de saúde, bem remunerados. ${ }^{9}$

Uma das questões que este momento crítico apresenta é a mudança do olhar do cuidado binário, sustentado na relação usuário-profissional, para uma visão coletiva de saúde da população ${ }^{10}$. Dito de outra forma, passa-se a buscar o alcance de estratégias de ação que impactem positivamente para o maior grupo de pessoas, ao invés de pensar individualmente ações positivas para uma única pessoa. Deslocase a ética individual para a ética coletiva ${ }^{11}$. Como o objetivo primordial da saúde coletiva é melhorar a saúde das populações e não dos indivíduos, entram em cena questões éticas que englobam o olhar coletivo sobre o individual' ${ }^{12}$. 
Entretanto, cabe ressaltar que os tratamentos regulares de saúde precisam ser mantidos e, portanto, os dois olhares não podem ser esquecidos. Principalmente refletindo sobre os critérios para alocação de recursos humanos e em grupos de usuários que precisam de atendimento e tratamento regular, como indivíduos portadores de doenças renais, reumatológicas, câncer, entre outros. Neste tocante, o Hasting Center dos Estados Unidos define o necessário equilíbrio de duas fontes de autoridade moral, a saber: 1- a ética clínica com o dever de cuidar como essencial para os cuidados de saúde, como obrigação moral e legal e; 2- ética de saúde pública com o dever para promover a igualdade moral entre as pessoas e a equidade (justiça em relação à necessidade na distribuição de riscos e benefícios na sociedade $)^{13}$.

Em situações de catástrofe, existe uma obrigação moral de organização e planejamento voltadas ao estabelecimento de critérios de alocação de recursos, quando houver escassez dos mesmos ${ }^{14}$. Estes critérios devem ser eticamente defensáveis, já que a técnica é moral dependente e, portanto, devem ser pautados em pilares clínicos, técnicos e éticos para que seja assegurado o atendimento ao maior número de pessoas ${ }^{15}$.

É importante ressaltar que todos os pilares devem ser igualmente visitados e analisados com pesos iguais, para que atinjam a máxima de salvar o maior número de vidas. Apesar da compreensão que nenhum protocolo será "eticamente perfeito e que alcance uma universalidade moral"16. Defende-se a ideia de um protocolo que seja, igualmente, justo para todos os indivíduos e pautados em princípios éticos. Estes, os princípios éticos, devem responder a questões centrais, a saber: quem tem direito ao recurso?; quais os motivos que justificam uma pessoa ser prioridade no 
acesso ao recurso que pode salvar sua vida em detrimento de outra?; como devem ser tomadas as decisões de prioridades?; quem deve tomar estas decisões? ${ }^{17}$.

Diante do que foi apresentado, este artigo objetiva analisar, os protocolos de alocação de recursos escassos propostos, como no apresentado pela Associação Médica Intensivista Brasileira (AMIB), apontando as consequentes fragilidades e desigualdades de acesso a tratamento da população em geral. Para tal, inicia-se com uma breve análise dos princípios que sustentam os protocolos e, a partir destes, as recomendações e critérios de estabelecimento de alocação de recursos escassos durante a pandemia. Após esta apresentação, será apontado as questões críticas que alargam as desigualdades e injustiças à população brasileira. Por fim, trazemos as considerações finais, não com uma conotação conclusiva, mas como apontamentos com possibilidades de revisão

\section{O Pilar Ético dos Protocolos}

A exemplo do ocorrido em outros países, a escassez dos recursos de saúde e as necessidades, cada vez mais crescentes do número de pacientes graves com COVID-19, que precisam de leitos de terapia intensiva, levam à situações críticas por conta dos recursos insuficientes diante a alta demanda de pacientes. Estas situações críticas e emergenciais demandam das entidades ligadas à área da saúde o desenvolvimento de protocolos para o "estabelecimento das melhores práticas para a otimização da utilização dos meios e recursos disponíveis"18.

Os protocolos são atravessados por questões que demandam reflexões sobre seus princípios/pressupostos. As tomadas de decisões implicam escolhas que são regidas por determinados valores e princípios. Quem deverá receber a assistência de respiradores ou não? Em caso de existir somente um respirador e dois ou mais 
pacientes igualmente graves, quem será o escolhido? Que critérios serão estabelecidos para subsidiar as escolhas?

Para o estabelecimento das decisões técnicas e clínicas dos protocolos, devese ter uma solidez ética, com princípios validados com pretensão de universalidade para justificar as escolhas difíceis que a alocação de recursos em tempos de pandemia requer. Vale ressaltar que, os protocolos, em geral, apontam diversos princípios para sustentarem seus critérios e recomendações, como por exemplo: dignidade humana, beneficência, justiça, autonomia, solidariedade e vulnerabilidade.

A Sociedade Brasileira de Bioética ${ }^{19}$ recomenda que a dignidade humana deve ser "o fundamento maior", já que resguarda as pessoas de quaisquer atos discriminatórios e de desvalorização. Para Santoni et al. ${ }^{20}$, a dignidade humana estaria associada a direito igualitário a triagem, transparência, acesso à informação e atendimento. A esse respeito, a $\mathrm{AMIB}^{21}$ acrescenta que nenhum grupo social deve ter maiores benefícios que outros, de maneira desproporcional.

A beneficência sustenta a recomendação de maximização de benefícios ${ }^{22}$. A Sociedade Espanhola de Medicina Intensiva, Crítica e Unidades Coronarianas ${ }^{23}$ ressalta a importância de desenvolver estratégias proativas em fases, objetivando aproveitar ao máximo os recursos limitados e mitigar os danos às pessoas. Apesar da $\mathrm{AMIB}^{24}$ não explicitar a beneficência, em seus pilares éticos, ressalta que a possibilidade de benefício do usuário deve ser um critério a ser utilizado.

O princípio de justiça, segundo a Sociedade Espanhola de Anestesiologia ${ }^{25}$, é justificado pela natureza peculiar da situação. Mas os olhares sobre este princípio parecem trazer algumas diferenças significativas e que são refletidas nas recomendações dos protocolos. Emanuel et $\mathrm{al}^{26}$ apontam que deve-se tratar 
igualmente a todos, recompensando o valor instrumental e dar prioridade aos mais pobres no atendimento. Já Duhnham, Rieder, Humbyrd ${ }^{27}$, a Sociedade Espanhola de Anestesiologia ${ }^{28}$, e a Sociedade Brasileira de Bioética ${ }^{29}$ trazem a indicação do uso justo e equitativo de recursos. $\mathrm{A} \mathrm{AMIB}^{30}$ ressalta a transparência e clareza, visando o esclarecimento de todos, como um aspecto bioético imprescindível para a condução justa da triagem.

Dentre as situações, a autonomia do paciente é algo valorável. No entanto, a autonomia limitada, se é que podemos pensar em limitação de autonomia, é colocada como "restrição de liberdade"31. A restrição de liberdade, segundo a autora, é defendida pela obrigatoriedade de exames de saúde para as pessoas sintomáticas, e justificam-se por ainda não termos um tratamento eficaz para a COVID-19. Ressalta ainda, que esta restrição deve estar associada a não violação de direito à privacidade e dignidade da pessoa humana. Apesar do protocolo da AMIB $^{32}$ apresentar como princípio a autodeterminação, a oferta de cuidados deve ser acordada no binômio equipe - usuário/família. E a vontade do usuário deve ser considerada, mas enfatiza que esta pode não ser acatada, restringindo sua autonomia de decisão. O que implica em autonomia limitada.

A solidariedade é apontada pelas Sociedades Espanhola de Anestesiologia (2020) e Brasileira de Bioética, Conselho de Bioética de Nuffield (2020) e Rede de Bioética da América Latina e do Caribe - UNESCO(2020) e Rubio (2020).Segundo a Rede de Bioética da América Latina e do Caribe - UNESCO ${ }^{33}$, gestos de solidariedade incluem deixar os comportamentos individualistas, reforçando que "é hora de mostrar atitudes éticas que transformem, para melhor, as pessoas e as sociedades, na visão de uma cidadania ativa que promova a plena validade da 
igualdade e dos direitos de todos". A mesma deve ser exercida, segundo o Conselho de Bioética de Nuffield ${ }^{34}$, desde o nível internacional até o nível individual, incluindo governos, empresas e indivíduos. Isso pode ser concretizado na organização de recursos, pela necessidade de uma contingência nacional, onde a solidariedade prevaleça entre os centros de saúde 35 .

$\mathrm{A} \mathrm{AMIB}^{36}$ inclui a vulnerabilidade como princípio balizador de seu protocolo, já que todos são vulneráveis, sejam os pacientes, profissionais de saúde e população em geral. Este princípio sustenta a manutenção de algum tipo de cuidado aplicado a todos os indivíduos, não os abandonando.

\section{O Pilar Clínico/Técnico dos Protocolos}

A primeira questão a ser levantada é por quem e com que fundamentos os critérios são estabelecidos? De modo geral, para tratamento em terapia intensiva os mais comuns são o modelo diagnóstico, o modelo de parâmetros objetivos e o modelo de priorização. No modelo diagnóstico, o profissional de saúde se remete a uma lista de patologias que demandam tratamento intensivo. No modelo de parâmetros objetivos, alguns parâmetros laboratoriais ou fisiológicos determinam a internação e alocação em leitos de UTI. No modelo de priorização, os pacientes são selecionados segundo um sistema de triagem, que prioriza pacientes conforme suas necessidades e chances de recuperação. Este último modelo é o que, prioritariamente, tem sido usado durante a pandemia do COVID-19. Um sistema de pontuação é estabelecido para estabelecer a alocação dos recursos. Neste sistema, os valores éticos passam a ser subjugados em uma escala numérica. Os pontos determinam quais vidas valem mais que as outras, tendo como categoria suprema o valor instrumental, de utilidade. Resta-nos refletir quais valores estão por trás dos números. 
O propósito dos critérios é ter definido de antemão os caminhos possíveis para as tomadas de decisões difíceis no âmbito da saúde quando se está diante de escolhas que implicam a vida e a morte. Escolher quem tem o direito de viver é uma incongruência sórdida. Qual vida vale mais que a outra? Isso é uma questão que demanda grande reflexão. A maioria dos protocolos, mesmo com algumas características peculiares, adotam basicamente a mesma conduta. Diante a calamidade, o apelo vai para a quantificação. Salvar o maior número de indivíduos. É preciso otimizar os recursos, não desperdiçar.

Então a regra é usá-los para quem tem maiores chances de se salvar, quem irá desocupar um leito e um respirador mais brevemente deve ter vantagem. Assim a "fila" anda e todos acham que fizeram a melhor escolha, pois a justificativa está nos números. É o poder da quantificação que se sobressai e os valores éticos se resolvem em uma escala numérica. Simples, se não houvesse tantas injustiças por trás dos números e das verdadeiras razões que perpassam as tomadas de decisões.

A exemplo do sistema de priorização temos inúmeros protocolos nacionais e internacionais. A seguir, é proposto uma visita aos protocolos da University of Pittsburgh, que tem sido um modelo de utilização para vários países e como exemplo no Brasil, da AMIB.

A recomendação do Protocolo de Pittsburgh baseia-se em três etapas. A primeira refere-se à criação de equipes de triagem, a segunda aos critérios de alocação de respirador e leito de UTI e a terceira sobre a reavaliação para continuidade dos cuidados intensivos e respirador. Na etapa de triagem é proposto uma equipe de triagem diferente da que presta o cuidado ao paciente. $\mathrm{Na}$ etapa de critérios para alocação dos recursos durante emergências de saúde pública o objetivo 
principal da estrutura é a maximização dos benefícios, elegendo pacientes que atendam aos critérios estabelecidos, atribuindo uma pontuação de prioridade usando uma escala de 1 a 8 . As pontuações mais baixas indicam maior probabilidade de benefício de cuidados intensivos e as mais altas não são prioridade no atendimento e podem ser indicadas para cuidados paliativos.

Os pontos prioritários, que definem quem pode ou não receber os recursos de saúde, são estabelecidos de acordo com a maior probabilidade de recuperação dos pacientes, avaliados com uma medida objetiva e validada sobre as condições de saúde, por exemplo, a pontuação do SOFA e menor acometimento de comorbidades. A pontuação bruta de prioridade pode ser codificada em três cores estipulando alta, intermediária e baixa prioridade. Caso exista empate entre pacientes com igual pontuação, será considerado o ciclo de vida para desempate, com prioridade para pacientes mais jovens, que tiveram menos oportunidade de viver todos os estágios da vida. Além disso, os profissionais de saúde, principalmente aqueles que dedicam atendimento direto a pacientes em cuidados intensivos, também são considerados prioridade.

Na terceira etapa do protocolo é referido sobre a necessidade de triagem para reavaliação para a verificação de continuidade de fornecimento contínuo de cuidados intensivos. O grupo de triagem conduzirá reavaliações periódicas de todos os pacientes que recebem serviços de cuidados intensivos para averiguar a evolução do quadro da doença e da gravidade da crise. A reavaliação deve ser usada para quantificar as alterações nas condições dos pacientes a partir de recálculo dos escores de gravidade da doença, avaliação de novas complicações e recolhimento da opinião dos médicos. Os pacientes que apresentarem melhora continuarão a 
receber cuidados intensivos, os que apresentarem baixa chance de sobrevivência terão os cuidados intensivos descontinuados e passarão a cuidados paliativos.

A proposta da $\mathrm{AMIB}^{37}$, é construída conjuntamente pela a Associação Brasileira de Medicina de Emergência (ABRAMEDE), pela Sociedade Brasileira de Geriatria e Gerontologia (SBGG), e pela a Academia Nacional de Cuidados paliativos (ANCP). Na segunda edição do protocolo se aproxima do sistema de pontos prioritários dos acadêmicos de Pittsburgh. Para os proponentes da proposta de desenvolvimento de um protocolo de triagem, que identifique quais pacientes teriam prioridade na alocação de recursos em esgotamento devido a pandemia de COVID19, não é um projeto meramente técnico, mas que considera os aspectos éticos e legais.

A AMIB e a ABRAMEDE editaram o primeiro protocolo intitulado Protocolo AMIB de alocação de recursos em esgotamento durante a pandemia por COVID-19, em abril do corrente ano. A urgência na edição justificava-se pela necessidade de poupar os profissionais de saúde da responsabilidade de tomar decisões difíceis, podendo comprometer sua saúde mental e, em consequência, sua capacidade laboral $^{38}$. No primeiro, os aspectos organizacionais de um protocolo foram superficialmente tocados. No segundo este aspecto foi mais desenvolvido, contribuindo nas ações administrativas em tempos de COVID que impedissem ou postergassem o momento das escolhas difíceis.

O primeiro protocolo incluía a pontuação da idade, que era estratificada em faixas etárias, e quanto maior a idade, maior a pontuação. Os dois protocolos também enfatizam que os usuários que não foram selecionados para ocupar a vaga de CTI 
devem "continuar recebendo os demais tratamentos não racionados quando clinicamente apropriado".

São utilizados "níveis de prioridade", especificados na Resolução CFM 2156/2016, que estabelece os critérios para indicação de admissão ou de alta em unidades de Terapia Intensiva ${ }^{39}$. Os níveis de prioridade utilizam como critérios, a necessidade de intervenções de suporte à vida/ monitorização intensiva, probabilidade de recuperação e limitação do suporte terapêutico. A escala vai de 1 a 5, onde o 1 é o que tem alta probabilidade de recuperação, sendo este critério escalonado até 4 , e 5 é o mais restritivo à suporte terapêutico, sendo considerado caso de terminalidade da vida, que poderiam excepcionalmente serem indicados, como por exemplo, futuros doadores de órgãos. Neste caso os usuários do Serviço de Nefrologia estariam entre a prioridade 3 e 4.

Nestes protocolos são utilizados critérios médicos e assistenciais, de preferência objetivos, utilizando instrumentos validados internacionalmente que se juntam em um grid de pontuações. No entanto, todos estes critérios, para serem eticamente defensáveis, devem ser "claros, transparentes, tecnicamente bem embasados, eticamente justificados e alinhados ao arcabouço legal brasileiro"40.

O segundo protocolo foi divulgado em maio do mesmo ano, trazendo contribuições de outros profissionais da área da saúde e do direito, visando torná-lo mais representativo dos valores e leis brasileiras ${ }^{41}$. Uma das mudanças foi a retirada da idade, como um dos critérios de triagem, justificado pelo cunho discriminatório e inconstitucional.

Neste segundo, passam a valer os seguintes itens de avaliação: SOFA (Sequential Organ Failure Assessment) simplificado, comorbidades graves, com 
expectativas de sobrevida menor que 1 ano, sugerindo o uso do instrumento SPICTBR (Supportive and Palliative Care Indicators Tool) e ECOG. O SOFA é um instrumento que atribui escores que variam de 1 a 4 , para avaliar o grau de comprometimento dos sistemas cardiovascular, respiratório, hematológico, neurológico e renal ${ }^{42}$.

O SPICT-BR é um instrumento que avalia a deterioração do corpo, com indicadores clínicos das condições avançadas e progressivas, levando em consideração câncer, demência/fragilidade; doença neurológica; doença cardiovascular; doença respiratória, doença renal e doença hepática.

O ECOG (Eastern Cooperative Oncology Group) é um medida de funcionalidade, analisada através de uma escala que quantifica a capacidade funcional física e de independência e autocuidado. Quanto pior a funcionalidade, menor sua reserva fisiológica e piores os desfechos clínicos. A escala pontua de 1 a 4, sendo um o paciente completamente ativo que desenvolve todas as tarefas independentemente até o totalmente dependente, estando confinado ao leito.

Quanto menor é a pontuação de um paciente, maior será a sua prioridade de alocação de recursos escassos. Aqueles com melhores condições de saúde global têm prioridade. Além disso, quanto maior o número de pacientes a serem triados, maior será a expectativa de empates nas pontuações e, por essa razão, é incluído sugestões de critérios de desempate, o qual é direcionado para a questão da idade/ciclo de vida.

Dos três instrumentos, dois avaliam clinicamente os candidatos a vaga do CTI, pautando-se exclusivamente nas condições dos sistemas e um avalia a capacidade funcional do indivíduo, o que pode sugerir que os previamente saudáveis poderão ter maiores chances de tratamento dentro da terapia intensiva. Assim como os usuários 
mais novos, já que os idosos tendem a ter uma deterioração dos sistemas. Esta questão soma-se a orientação do protocolo para que todos os usuários que necessitem de um leito de CTI, devem ser igualmente submetidos aos critérios previamente definidos, independente se a doença a ser tratada seja COVID-19 ou não43.

Os autores do protocolo da AMIB alegam que este protocolo possui vantagens e limitações. Primeiramente, apontam que faz parte da responsabilidade de profissionais e do poder público o preparo para a possibilidade de esgotamento de recursos. De acordo com recomendações e diretrizes de sociedades médicas internacionais, o estabelecimento de um protocolo de alocação de recursos em esgotamento é uma exigência que faz parte do preparo para uma situação de pandemia em que existe a possibilidade de que as medidas de contingenciamento não sejam suficientes para lidar com a demanda aumentada de pacientes graves. Resta-nos saber se e como estas recomendações se efetivam na prática, principalmente em um país com tantas dificuldades estruturais, culturais e políticas, como é o caso do Brasil.

\section{Uma visão crítica sobre protocolos de alocação de recursos escassos durante a pandemia por COVID-19}

Os protocolos são necessários em um situação de crise como a que se apresenta na pandemia por COVID-19. No entanto, nesta situação, mesmo catastrófica, é fundamental refletir de modo crítico sobre os princípios e critérios que sustentam a construção dos protocolos. Não é porque vivemos uma catástrofe que tudo será permitido sem consideração ao propósito ético.

\section{1- Um retomada ao Comitê dos Deuses de Seattle - Quem participa da decisão?}


A AMIB, nos três protocolos que editou, reuniu profissionais da saúde e do direito para definição dos protocolos. Porém, em nenhum dos protocolos foram refletidas estratégias para inclusão de associações de usuários das mais diversas especialidades médicas. Pensando em pandemia, e na modificação do olhar da ética clínica para a ética coletiva, deveria valorizar-se a estrutura do consenso, onde governantes e as instituições hospitalares poderiam criar conselhos para definição de alocação de recursos ${ }^{44}$. O Brasil é em um país que tem conselhos de saúde nas três esferas, com a participação efetiva de usuários do sistema de saúde, de acordo com a Lei no 8142, de 28 de dezembro de 199045. Este coletivo favorece a corresponsabilidade de todos. Deste modo, diferentes pontos de vista são colocados e evita-se vieses típicos de interpretações individuais ${ }^{46}$.

A falta desta pluralidade, implica em questões éticas. Um bom exemplo desta situação aconteceu há aproximadamente 40 anos atrás, e que atualmente é reconhecido como um dos marcos da bioética, quando foi desenvolvido o shunt arteriovenoso por Belding Scribner, na década de 60. A invenção de algo que pudesse permanecer no braço do doente renal, realizada por Belding Scribner, aumentava a demanda de usuários que poderiam utilizar a máquina e usufruir de seus benefícios para o tratamento de sua doença. A máquina para tratamento renal substitutivo já existia, sendo a primeira construída em 1912. Entretanto, tinha o inconveniente de cada vez que o paciente era ligado a máquina, uma artéria ou veia tinha que ser aberta, tornando limitada sua utilização.

Havia, porém, apenas uma máquina de hemodiálise para ser utilizada. Diante do conflito moral para sua utilização, tornou-se urgente o estabelecimento de um comitê que definiria os critérios morais para julgamento das pessoas que seriam 
selecionadas para o tratamento e, diante desta possibilidade, teriam aumento das chances de sobrevida ${ }^{47}$. Ressalta-se que este conflito data da década de 60 , e nos parece tão atual diante das pandemias de Influenza, Ebola, e mais recentemente, a COVID-19. Estabeleceu-se, então, o "Comitê de Políticas de Admissão do Seattle Artificial Kidney Center"48, apelidado mais tarde de Deuses de Seattle ou Comitê de Vida e Morte, com os seguintes participantes: um pastor, um advogado (o presidente da comissão $)^{49}$, uma dona-de-casa, um líder sindicalista, um funcionário público, um banqueiro e um cirurgião. A decisão de não ter diretamente, a presença de muitos médicos na comissão pautava-se no sofrimento gerado ao profissional diante da possibilidade de ter que decidir sobre seus próprios clientes ${ }^{50}$. A jornalista da LIFE, ressalta que, em relação a composição do comitê, "significava a aceitação do princípio de que todos os segmentos da sociedade, não apenas a fraternidade médica, deveriam compartilhar o ônus da escolha de quais pacientes tratar e quais deixar morrer. Caso contrário, a sociedade estaria forçando apenas os médicos a brincar de Deus" 51 .

As críticas, à época, versavam sobre a qualificação dos membros, visto que a maioria era classificado como leigos, assim como a ausência de critérios relativos ao estado da doença ou da expectativa de vida do paciente durante o tratamento. $\mathrm{Na}$ atualidade, vive-se algo similar ao "Comitê de Deuses", só que desta vez qualificados tecnicamente, mas faz-se presente a monopolização das decisões.

Conforme Dias e Gonçalves ${ }^{52}$ apontam, este debate deveria ser ampliado recebendo outras vozes. A decisão de realizar triagem singular dos casos, como proposto pela AMIB parece apropriada, mas as equipes de avaliação para os cuidados intensivos devem ser compostas de, pelo menos, três tipos de profissionais. Dois médicos(as), um outro profissional de saúde e um eticista, que deve ser incluído. 
Antes que as decisões sejam tomadas é importante a transparência e clareza sobre quais vozes se fazem presentes no debate e sobre todas as questões que subjazem as tomadas de decisões.

Silva et $a^{53}$ apontam quatro razões para que a consulta pública seja realizada:

1- uma resposta efetiva de saúde pública, depende de informação e engajamento da população; 2- garante a responsabilidade democrática dos formuladores de políticas governamentais; 3- melhora as decisões sobre as políticas públicas; 4- estabelece confiança, o que é essencial para que as pessoas possam apoiar as decisões e ajudem a promover respostas oportunas e eficazes.

No estudo realizado no Canadá sobre a perspectiva da população canadense quanto ao estabelecimento prioritário de recursos de UTI na pandemia de influenza, os participantes reconheceram que decisões difíceis devem ser tomadas em tempos de pandemia. Se preocupavam tanto com os valores e critérios que definiriam as prioridades, como também com quem e como estas decisões seriam tomadas. Por isso, reconheciam a importância de um comitê para deliberar, formado não apenas por médicos, evitando que uma pessoa ou um grupo restrito assuma a carga moral de deixar viver ou morrer ${ }^{54}$.

Como bem colocado por Rajan $\mathrm{D}$ et $\mathrm{a}^{55}$, o vírus HIV nos ensinou que a população, comunidade e sociedade civil devem integrar a solução do problema a ser enfrentado, o que corrobora a realidade brasileira. $\mathrm{O}$ autor enfatiza que é imperioso reconhecer a multidimensionalidade dos efeitos e necessidades da sociedade, consultando as várias áreas do conhecimento e não apenas da saúde. Incluindo "as vozes daqueles que correm o risco de serem deixados por trás precisam ser ouvidos".

Alguns colegiados e associações recomendam a criação de comitês em que sejam incluídos bioeticistas, entre eles o Colégio Americano de Cirurgiões, a Sociedade Espanhola de Anestesia e UNESCO. A esse respeito, Dias e Gonçalves ${ }^{56}$ a ampliam ressaltando que a única saída é o convite ao debate, não apenas à 
eticistas, mas a toda a sociedade. Referencia a criação imediata de um Comitê Bioético Nacional composto interdisciplinarmente, e que possa assessorar as decisões estratégicas neste momento tão grave. O debate precisa ser público para que o peso das escolhas de quem deve viver e quem pode morrer, mais uma vez, não recaia sobre os segmentos historicamente mais fragilizados, invisibilizados e vulnerabilizados.

\section{2- A desigualdade no peso atribuído aos critérios: a falácia dos princípios}

De acordo com Dias e Gonçalves ${ }^{57}$, quanto aos critérios para escolha, algumas questões são importantes de serem discutidas. São elas: a falta de neutralidade, repúdio ao sorteio e falso dilema. Em um primeiro patamar de discussão é levantado que os critérios não são neutros, nem auto evidentes. Por essa razão, é necessário justificá-los à luz de seus valores e crenças e não como algo definido em uma falsa clareza de propósito.

Para as referidas autoras, é importante indagar: Por que seria mais justo, por exemplo, salvar antes um profissional de saúde ou alguém mais jovem? No primeiro caso, a justificativa seria de que tais profissionais poderiam voltar a ajudar salvar outros indivíduos. Mas há por trás o argumento, que, no mínimo, adota uma visão instrumental do que seria o valor da vida desses mesmos indivíduos.

O critério de idade, explicitamente colocado no primeiro protocolo da $\mathrm{AMIB}^{58}$, mas retirado na segunda edição, ou dito de outra forma, o critério de "equalizar as oportunidades de se passar pelos diferentes ciclos da vida", implica o argumento de que pessoas que viveram menos anos devem ter o direito de atingir os ciclos de vida posteriores. Quais as crenças e valores estariam por trás deste argumento? Em que medida mais tempo de vida pode ser algo em si valorável, e que pode ser estabelecido 
como um "direito" que coloca alguns diante os outros? Será o "tempo de vida" um bem justificável e que é preciso distribuí-lo de forma equitativa? Para Dias e Gonçalves ${ }^{59}$, o ato de atribuir um valor moral formal ao "tempo ou ciclo de vida" desconsidera o conteúdo concreto das vidas vividas, ou seja, o valor que cada indivíduo atribui a suas escolhas, realizações e projetos de vida. "O direito a viver mais anos, de alguns, não pode se sobrepor ao direito de continuar vivendo, de outros".

A questão do idoso, apesar de na nova edição não ser claramente colocada, se coloca em questão. Com a idade, existe uma deterioração fisiológica dos sistemas funcionais e a capacidade física é menor, levando algumas vezes a algum grau de dependência e dificuldades em se auto-cuidar. Se todos os instrumentos avaliam as condições clínicas dos sistemas do corpo humana e a capacidade funcional, o idoso com certeza, já sairá em desvantagem em relação a uma pessoa mais nova. Portanto, apesar de não explicitamente, o ageísmo é um valor no protocolo, reforçando a instrumentalização da pessoa humana, enquanto produtor e consumidor. Está em desalinho com o princípio da dignidade humana, que sustenta este protocolo. Diante disso, infere-se que o critério idade/ciclo de vida pode ter consequências altamente questionáveis que se confrontam com outros temas importantes à Bioética.

Assim como no ageísmo, parece haver, a partir dos instrumentos estabelecidos, uma culpabilização do usuário por não ser saudável. Os instrumentos são legais e validados, sendo apresentados como justos, já que todos serão terão igual possibilidade de serem submetidos aos mesmos critérios. Neste sentido a igualdade seria o justo, mas considerando que as desigualdades sociais com o acesso aos bens de saúde, educação, saneamento básico, entre outros, em 
associação aos marcadores sociais de diferença, associado a raça, etnia, gênero, orientação sexual, classe social, o valor de igualdade valeria para nossa realidade? Se a igualdade for considerado justa, estaremos culpabilizando as pessoas por não terem saúde. Cabe esta culpa e a pessoa deve arcar com as consequências de não ser saudável? Dita de outra forma: a quem cabe esta culpa por termos pessoas saudáveis e outras nem tanto?

A nota técnica número 11, do Núcleo de Operações e Inteligência em Saúde ${ }^{60}$, aponta claramente que a igualdade está aumentando as bordas das desigualdades. A análise da variação da taxa de letalidade da COVID-19 no Brasil, considerando as variáveis socioeconômicas, apontou que: (1) os pretos e pardos apresentam um número maior de óbitos, em relação aos brancos, em todas as faixas etárias; (2) quanto menor o nível de escolaridade, maior a letalidade do COVID - neste marcador específico, os pretos e pardos apresentam um maior percentual de óbitos em todos os níveis de escolaridade e; (3) os municípios com baixo ou médio IDHM(Índice de Desenvolvimento Humano Municipal) praticamente dobram em municípios com IDH muito alto - mesmo nos municípios com o referido índice médio e alto, o maior percentual de óbitos ainda é de pretos e pardos.

Conforme ressalta o boletim informativo do Observatório de Direitos Humanos,

O enfrentamento à pandemia revela, com muita força, a falência de um modelo social, econômico e de bem-estar, especialmente na efetivação de direitos humanos e acesso aos serviços essenciais pelas populações mais vulneráveis. Para a população de menores rendimentos, com baixos indicadores sociais, para as pessoas, em sua maioria mulheres, que estão na linha de frente dos serviços de saúde, e as mais vulneráveis à ação do mercado e do Estado violentador, a ocorrência da pandemia tem agudizado as desigualdades sociais e violações de direitos já existentes. ${ }^{61}$ 
Diante disso, considerar o princípio da vulnerabilidade, apenas associado ao risco de adoecimento por COVID-19, não atende a realidade brasileira. O princípio de vulnerabilidade deve estar relacionado a vida das pessoas em suas múltiplas facetas, considerando às condições das pessoas de se protegerem e de não adoecerem, incluindo-se aí a avaliação das especificidades, buscando sim, a equidade no atendimento da população. O princípio da "vulnerabilidade universal" associado à igualdade, só perpetua as disparidades tão presente na realidade brasileira. $\mathrm{Na}$ verdade, as mortes pelo COVID-19 fazem refletir as desigualdades de classe e raça que estruturam a sociedade brasileira desde o início dos tempos. Por que seria diferente agora? As escolhas sempre implicam em vidas que valem mais que outras, e essa escolha subjaz questões arraigadas nas entranhas de uma sociedade que não prima por justiça e vida digna a todos os cidadãos igualitariamente. As estratégias sanitárias não desvinculam-se do contexto econômico, político e social em sua amplitude.

Há muitas vidas que não possuem o mínimo para a subsistência, que vivem em condições precárias, não devemos dar direito ao uso de uma pandemia, da proporção como a que assola o mundo neste ano de 2020, para validar padrões eugenistas a partir de uma necropolítica racista, homofóbica, sexista e elitista. A única validade para o momento é incluir com urgência na pauta de discussão os temas de desigualdade social e a precariedade das condições de vida, que são impostas à grande maioria dos cidadãos brasileiros.

Ademais, os critérios estabelecidos, ainda que por eticistas, ou outros grupos, não podem subsidiar a incorporação de certas mortes, seja de idosos ou indivíduos pertencentes à população de risco, como consequência inevitável da pandemia. Não 
se pode naturalizar se utilizando de uma situação tão grave, as escolhas sociais, políticas e econômicas realizadas pela sociedade e seus governantes há séculos. A sugestão deve ser a de um redimensionamento crítico, e não pontual, das estratégias para enfrentamento da pandemia ${ }^{62}$.

\section{3- A experiência não se sobrepõe à realidade: aprender e não copiar}

Uma outra questão é a necessária avaliação e estabelecimento de diretrizes específicas para atendimento da nossa realidade e não repetir protocolos de outros países. O conhecimento e expertise dos países que viveram a pandemia antes do nosso, deve servir de base e experiência para a definição dos nossos critérios. É vital considerar os vários modelos de sistemas de saúde diferentes, formas políticas de abordar as desigualdades sociais e econômicas, e as realidades de cada localidade. Inclusive no que se refere ao direito básico à saúde, e suas interfaces com os determinantes sociais de saúde, para buscar formas justas, ou menos injustas de estabelecer os critérios.

\section{Considerações Finais}

Para finalizar as considerações propostas neste artigo, não no intuito de encerrar uma discussão, mas com vistas a abrir ao debate, algumas ponderações são importantes diante à necessidade de utilização dos protocolos e da forma como são constituídos. Para inaugurar o debate, a partir de tudo que foi colocado até aqui, uma questão se sobressai: Seria possível algum protocolo que fosse pautado por questões éticas e de justiça distributiva?

Evidentemente, diante da escassez de recursos, são necessárias verificações no sentido de se identificar se tal escassez é decorrente de injustiças ou iniquidades 
na distribuição de recursos. Na correção de tais injustiças repousa talvez o principal foco de atuação por parte da sociedade e seus cidadãos. No entanto, independente da situação existente, o profissional de saúde na linha de frente de atendimento pode se deparar com um fato: a escassez de recursos para um grande número de indivíduos necessitados deles. Portanto parece que a existência de algum tipo de protocolo é um passo importante na minimização de injustiças. A possibilidade contrária, isto é, a não utilização de protocolos implicaria em situações absurdamente imorais, tais como a chegada do recurso escasso por ordem de chegada ou mesmo sorteio (já referido anteriormente) ou ainda a não oferta do recurso para ninguém.

Se a necessidade de um protocolo parece ser ponto ético e justo na definição da assistência aos doentes, que elementos poderiam ser elencados em seu desenvolvimento?

1) Criação do protocolo a partir dos concernidos morais envolvidos com o problema.

Um protocolo que fosse desenvolvido a partir de um grupo específico (apenas profissionais de saúde, ou pacientes ou gestores, por exemplo) correria enorme risco de ser contaminado por uma visão unilateral que não contemplasse a escuta de todos os demais. Em última análise, se os profissionais de saúde conhecem mais determinados aspectos técnicos (por exemplo, o comprometimento pulmonar associado à COVID), ninguém melhor do que os familiares de pacientes para externar o sofrimento associado com o distanciamento físico dos doentes e aos problemas de comunicação de notícias relacionadas. Questões de raça, gênero, classe social, para citar algumas, sempre foram fundamentais na maior ou menor vulneração dos indivíduos. A desconsideração de informações provenientes de qualquer grupo aqui 
poderia representar menosprezo por tais grupos ou até mesmo arrogância intelectual. A proposta de comissões ou comitês gerais com ampla inclusão em seus membros, conforme defendido por Dias e Gonçalves ${ }^{63}$ vem bem ao encontro dessa ideia, no sentido de minimização de possíveis injustiças na referida alocação.

2) Preferências de determinados grupos na alocação desses recursos

Diante de uma comissão plural, com a ponderação de razões públicas, poderá emergir a necessidade de priorização de algum grupo ou indivíduo. Políticas de discriminação positivas não são novidades no campo da justiça distributiva. As cotas raciais, por exemplo, tem se mostrado eficazes na redução de injustiças relacionadas à população negra ${ }^{64}$, na alocação de recursos ligados à educação. No entanto, são necessários dois pontos para tal discriminação, conforme aponta Dworkin ${ }^{65}$ : (a) que haja embasamento teórico, configurado em razões públicas que possam justificar tais preferências e (b) que haja apoio empírico na ratificação da eticidade dessas propostas.

Veja-se, por exemplo, a preferência que alguns protocolos ${ }^{66}$ pretendem dar a profissionais de saúde: de que uma vez curados, poderiam voltar à linha de frente para salvar mais vidas. Parece haver aqui enorme confusão. Por um lado não há garantia alguma de que tal profissional tenha condições de salvar alguém pós-alta: COVID tem se mostrado doença altamente incapacitante em situações de alta das Unidades De Terapia Intensiva ${ }^{67}$ e por outro, é necessário que se assuma pelo menos algum referencial utilitarista, na maximização no número de vidas potencialmente salvas (o que não é nada óbvio em se tratando de ética).

3) Não-preferência de determinados grupos na alocação 
Não é novidade a exclusão de determinados grupos na admissão em unidades de terapia intensiva, e poderia mesmo ser o caso de justiça bem aplicada em alguns. Tome-se, por exemplo, a internação de moribundos em tais unidades. Há consenso mundial ${ }^{68}$ de que tais pacientes falecem a caminho da unidade ou nos momentos iniciais em sua admissão. Isso acarreta a perda ou adiamento da possibilidade de vaga para outro paciente viável e com chance real de melhora com os cuidados intensivos (e muitas vezes, o tempo é fundamental nesses casos).

No entanto, se para o caso específico relatado parece haver apoio teórico e empírico, em outros casos isso parece inexistir. A situação dos idosos, por exemplo. Alguns protocolos defendem a não-priorização para este grupo etário, caso da ltália69. A idade avançada não é sinônimo de pré-morte. Há pacientes moribundos em qualquer faixa etária. Alguns trabalhos, como se vê em Azevedo ${ }^{70}$ apelam para um "ciclo de vida", onde o idoso já teria vivido muito e deveria ter seu direito a uma vaga transferido para outro indivíduo mais jovem. O equívoco aqui parece ser duplo: primeiramente pelo ancoramento de uma decisão ética à ideia de natureza, nem a medicina faz tomadas de decisão nessa base (a todo tempo, ela parece lutar mesmo contra a natureza). Parece não haver base sólida para uma ideia de uma natureza intrinsecamente boa. Em segundo lugar, para essa ideia ser levada a sério, ela precisaria ter no mínimo um grau de coerência (a mesma tese engendra a ideia de alguém de 30 anos ceder sua vez para alguém de 20 anos pelo fato de o indivíduo de 30 já ter vivido mais?).

Talvez mais importante do que apontar positivamente tal ou qual critério (o que certamente deverá existir nas bases de apoio acima citadas), a identificação de 
Diversitates Int. J. (ISSN: 1984-5073) - Vol. 12, N.2, Julho/Dezembro (2020), p. 131 - 162

critérios injustos e sua não-inclusão em protocolos seja etapa fundamental no processo de minimização de injustiças nesse tipo de alocação.

\title{
Referências:
}

\footnotetext{
${ }^{1}$ Sohrabi, Catrin et al. World Health Organization declares global emergency: A review of the 2019 novel coronavirus (COVID-19). International Journal of Surgery, 2020.

2 World HealthOrganization. Coronavirus disease 2019 (COVID-19)

Situation Report - 51 [OMS]. Publicado em 11/03/2020; acessada em 17/06/2020.

https://www.who.int/docs/default-source/coronaviruse/situation-reports/20200311-sitrep-51-covid19.pdf?sfvrsn=1ba62e57_10

${ }^{3}$ World Health Organization. Coronavirus disease (COVID-19) Situation Report - 148 [OMS]. Publicado em 16/06/2020; acessado em 17/06/2020. https://www.who.int/docs/defaultsource/coronaviruse/situation-reports/20200616-covid-19-sitrep-148-draft.pdf?sfvrsn=9b2015e9 2
}

\begin{abstract}
${ }^{4}$ Barreto C. Ministério da Saúde confirma primeira morte por coronavírus no Brasil [pbmed.com.br]. Publicado em 17/03.2020; acessado em 17/06/2020. https://pebmed.com.br/ministerio-da-saudeconfirma-primeira-morte-por-coronavirus-no-brasil/
\end{abstract}

5

Ministério da Saúde. acessado em 17/06/2020. https://covid.saude.gov.br/

Idoeta, PA. A matemática das UTIs: 3 desafios para evitar que falte cuidado intensivo durante a pandemia no Brasil [bbc.com]. 06 de abril de 2020; acessada em 17/06/2020.

https://www.bbc.com/portuguese/brasil-52137553

${ }^{6}$ https://www.bbc.com/portuguese/brasil-52137553 <acessada em 17/06/2020>

${ }^{7}$ BRASIL. Lei No 8142, de 28 de dezembro de 1990. Dispõe sobre a participação da comonidade na gestão do Sistema Único de Saúde (SUS) e sobre as transferências intergovernamentais de recursos financeiros na área da saúde e dá outras providências.

${ }^{8}$ Brasil. Instituto de Estudos Sócio Econômicos. O Brasil com baixa imunidade - Balanço do Orçamento Geral da União. 2020. Disponível em: https://www.inesc.org.br/wp-

content/uploads/2020/04/Balanco-OGU-Inesc.pdf

${ }^{9}$ Brasil. Instituto de Estudos Sócio Econômicos. O Brasil com baixa imunidade - Balanço do

Orçamento Geral da União. 2020 Acesso em: https://www.inesc.org.br/wpcontent/uploads/2020/04/Balanco-OGU-Inesc.pdf , p.50)

${ }^{10}$ Martins AA. Três obrigações bioéticas na resposta à Covid-19 e à escassez de recursos [ihu.unisinos.br]. www.ihu.unisinos.br/78-noticias/597569-tres-obrigacoes-bioeticas-na-resposta-acovid-19-e-a-escassez-de-recursos

${ }^{11}$ Dunham, AM E at al. A bioethical perspective for navigating moral dilemmas amidst the COVID-19 pandemic. The Journal of the American Academy of Orthopaedic Surgeons, 2020. 
Diversitates Int. J. (ISSN: 1984-5073) - Vol. 12, N.2, Julho/Dezembro (2020), p. 131 - 162

${ }^{12}$ Kass E Nancy E. An ethics framework for public health. American journal of public health, v. 91, n. 11, p. 1776-1782, 2001.

${ }^{13}$ The Hastings Center de EEUU. Ethical Framework for Health Care Institutions Responding to Novel Coronavirus SARS-CoV-2 (COVID-19). Guidelines for Institutional Ethics Services Responding to COVID-19. Managing Uncertainty, Safeguarding Communities, Guiding Practice.

${ }^{14}$ Martins AA. Três obrigações bioéticas na resposta à Covid-19 e à escassez de recursos [ihu.unisinos.br]. www.ihu.unisinos.br/78-noticias/597569-tres-obrigacoes-bioeticas-na-resposta-acovid-19-e-a-escassez-de-recursos

15

Satomi, E et al. Alocação Justa de Recursos de Saúde Escassos diante da Pandemia de COVID-19: Considerações Éticas . Einstein (São Paulo). 2020; 18: eAE5775. http://dx.doi.org/10.31744/einstein journal/2020AE5775;

RUBIO O, et al. Recomendaciones éticas para la toma de decisiones difíciles en las unidades de cuidados intensivos ante la situación excepcional de crisis por la pandemia por COVID-19: revisión rápida y consenso de expertos. Med Intensiva. 2020.

https://doi.org/10.1016/j.medin.2020.04.006

16

Versão um- Kretzer, L E et al. Protocolo AMIB de alocação de recursos em esgotamento durante a pandemia por COVID-19. Disponível em:

https://www.amib.org.br/fileadmin/user_upload/amib/2020/abril/24/Protocolo_AMIB_de_alocacao_de_ recursos_em_esgotamento_durante_a_pandemia_por_COVID-19.pdf

Versão dois - Kretzer, L E et al. Recomendações da AMIB (Associação de Medicina Intensiva Brasileira), ABRAMEDE (Associação Brasileira de Medicina de Emergência, SBGG (Sociedade Brasileira de Geriatria e Gerontologia) e ANCP (Academia Nacional de Cuidados Paliativos) de alocação de recursos em esgotamento durante a pandemia por COVID-19. Disponível em: https://www.amib.org.br/fileadmin/user_upload/amib/2020/abril/24/VJS01_maio

${ }^{17}$ Silva et al.: Priority setting of ICU resources in an influenza pandemic: a qualitative study of the Canadian public's perspectives. BMC Public Health 2012 12:241.

${ }^{18}$ Satomi, E et al. Alocação Justa de Recursos de Saúde Escassos diante da Pandemia de COVID19: Considerações Éticas . Einstein (São Paulo). 2020; 18: eAE5775.

http://dx.doi.org/10.31744/einstein_journal/2020AE5775;

${ }^{19}$ Cesari, M.; Proietti, M. COVID-19 in Italy: Ageism and Decision Making. COVID-19 in Italy: Ageism and Decision Making in a Pandemic.J Am Med Dir Assoc ; 21(5): 576-577,2020. P. 1)

${ }^{20}$ este é Azevedo, M E et al. Por um Debate sobre as Diretrizes Éticas para Alocação de Tratamento em UTI durante a Pandemia. 17 de abril de 2020, disponível em https://estadodaarte.estadao.com.br/amplo-debate-criterios-uti-coronavirus/. Último acesso em 18/06/2020.

${ }^{21}$ Versão um- Kretzer, L E et al. Protocolo AMIB de alocação de recursos em esgotamento durante a pandemia por COVID-19. Disponível em:

https://www.amib.org.br/fileadmin/user_upload/amib/2020/abril/24/Protocolo_AMIB_de_alocacao_de_ recursos_em_esgotamento_durante_a_pandemia_por_COVID-19.pdf 
Diversitates Int. J. (ISSN: 1984-5073) - Vol. 12, N.2, Julho/Dezembro (2020), p. 131 - 162

22 Emanuel, E. J.P. et al. Fair Allocation of Scarce Medical Resources in the Time of Covid-19. New England Journal of Medicine[2020]. <https://doi.org/10.1056/NEJMsb2005114>

${ }^{23}$ Sociedad Española de Medicina Intensiva, Crítica y Unidades Coronarias (SEMICYUC).

Recomendaciones generales relacionadas con las decisiones éticas difíciles y la adecuación de la intensidad asistencial / ingreso en las unidades de cuidados intensivos en situaciones excepcionales de crisis, 2020. Acesso em: https://semicyuc.org/wp-

content/uploads/2020/03/\%C3\%89tica_SEMICYUC-COVID-19.pdf

${ }^{24}$ Versão um- Kretzer, L E et al. Protocolo AMIB de alocação de recursos em esgotamento durante a pandemia por COVID-19. Disponível em:

https://www.amib.org.br/fileadmin/user_upload/amib/2020/abril/24/Protocolo_AMIB_de_alocacao_de_ recursos_em_esgotamento_durante_a_pandemia_por_COVID-19.pdf

${ }^{25}$ Sociedade Espanhola de Anestesiologia (2020) acesso em:

https://www.sedar.es/images/site/BIBLIOGRAFIA COVID-

19/Anestesia/DOC SEDAR Marco etico pandemia coronavirus.pdf

${ }^{26}$ Emanuel, E. J.P. et al. Fair Allocation of Scarce Medical Resources in the Time of Covid-19. New England Journal of Medicine[2020]. <https://doi.org/10.1056/NEJMsb2005114>

${ }^{27}$ Dunham, AM E at al. A bioethical perspective for navigating moral dilemmas amidst the COVID-19 pandemic. The Journal of the American Academy of Orthopaedic Surgeons, 2020.

${ }^{28}$ a Sociedade Espanhola de Anestesiologia (2020) acesso em:

https://www.sedar.es/images/site/BIBLIOGRAFIA COVID-

19/Anestesia/DOC SEDAR Marco etico pandemia coronavirus.pdf

${ }^{29}$ Sociedade Brasileira de Bioética. RECOMENDAÇÃO SBB No 01/2020. Aborda fundamentos e aspectos éticos no enfrentamento da pandemia COVID 19 no Brasil, incluindo a alocação de recursos e o uso igualitário das tecnologias em saúde. Defende a proteção aos mais vulneráveis e a imprescindibilidade do Sistema Único de Saúde (SUS), o direito ao acesso ao melhor tratamento para todos e o acesso igualitário a todos os leitos de UTI, públicos e privados. Recomenda princípios para definição de parâmetros a serem seguidos em situação de insuficiência destes. 2020.

${ }^{30}$ Versão dois - Kretzer, L E et al. Recomendações da AMIB (Associação de Medicina Intensiva Brasileira), ABRAMEDE (Associação Brasileira de Medicina de Emergência, SBGG (Sociedade Brasileira de Geriatria e Gerontologia) e ANCP (Academia Nacional de Cuidados Paliativos) de alocação de recursos em esgotamento durante a pandemia por COVID-19. Disponível em: https://www.amib.org.br/fileadmin/user_upload/amib/2020/abril/24/VJS01

${ }^{31}$ Salas, SP. Aspectos éticos de la epidemia del Coronavirus. Rev Med Chile. 2020; 148: 123-129

32 Versão Três- Dal-Pizzol, F(Org) Recomendações da Associação de Medicina Intensiva Brasileira para a abordagem do COVID-19 em medicina intensiva, 2020. Disponível em:

https://www.amib.org.br/fileadmin/user_upload/amib/2020/abril/13/Recomendaco_es_AMIB-atual.16.04.pdf, p.13)

${ }^{33}$ UNESCO. Declaração da Rede de Bioética da América Latina e do Caribe - UNESCO. 2020. http://www.sbbioetica.org.br/Noticia/706/Declaracao-da-Rede-de-Bioetica-da-America-Latina-e-doCaribe-UNESCO-divulgada-em-16032020-sobre-a-pandemia-de-COVID-19

${ }^{34}$ Nuffield Council. Ethical considerations in responding to the COVID-19 pandemic.2020 
Diversitates Int. J. (ISSN: 1984-5073) - Vol. 12, N.2, Julho/Dezembro (2020), p. 131 - 162

${ }^{35}$ RUBIO O, et al. Recomendaciones éticas para la toma de decisiones difíciles en las unidades de cuidados intensivos ante la situación excepcional de crisis por la pandemia por COVID-19: revisión rápida y consenso de expertos. Med Intensiva. 2020. https://doi.org/10.1016/j.medin.2020.04.006

${ }^{36}$ Versão três- Dal-Pizzol, F(Org) Recomendações da Associação de Medicina Intensiva Brasileira para a abordagem do COVID-19 em medicina intensiva, 2020. Disponível em:

https://www.amib.org.br/fileadmin/user_upload/amib/2020/abril/13/Recomendaco_es_AMIB-atual.16.04.pdf

${ }^{37}$ Versão dois - Kretzer, L E et al. Recomendações da AMIB (Associação de Medicina Intensiva Brasileira), ABRAMEDE (Associação Brasileira de Medicina de Emergência, SBGG (Sociedade Brasileira de Geriatria e Gerontologia) e ANCP (Academia Nacional de Cuidados Paliativos) de alocação de recursos em esgotamento durante a pandemia por COVID-19. Disponível em:

https://www.amib.org.br/fileadmin/user_upload/amib/2020/abril/24/VJS01

${ }^{38}$ Versão um- Kretzer, L E et al. Protocolo AMIB de alocação de recursos em esgotamento durante a pandemia por COVID-19. Disponível em:

https://www.amib.org.br/fileadmin/user_upload/amib/2020/abril/24/Protocolo_AMIB_de_alocacao_de_ recursos_em_esgotamento_durante_a_pandemia_por_COVID-19.pdf

${ }^{39}$ CONSELHO FEDERAL DE MEDICINA. Resolução 2156 de 2016. Disponível em:

https://portal.cfm.org.br/index.php?option=com_content\&view=article\&id=26557:2016-11-17-13-28-

$46 \&$ catid $=3$

40

Versão um- Kretzer, L E et al. Protocolo AMIB de alocação de recursos em esgotamento durante a pandemia por COVID-19. P. 3. Disponível em:

https://www.amib.org.br/fileadmin/user upload/amib/2020/abril/24/Protocolo AMIB de alocacao de recursos em esgotamento durante a pandemia por COVID-19.pdf

Versão dois - Kretzer, L E et al. Recomendações da AMIB (Associação de Medicina Intensiva Brasileira), ABRAMEDE (Associação Brasileira de Medicina de Emergência, SBGG (Sociedade Brasileira de Geriatria e Gerontologia) e ANCP (Academia Nacional de Cuidados Paliativos) de alocação de recursos em esgotamento durante a pandemia por COVID-19. P. 2. Disponível em: https://www.amib.org.br/fileadmin/user_upload/amib/2020/abril/24/VJS01

${ }^{41}$ Versão dois - Kretzer, L E et al. Recomendações da AMIB (Associação de Medicina Intensiva Brasileira), ABRAMEDE (Associação Brasileira de Medicina de Emergência, SBGG (Sociedade Brasileira de Geriatria e Gerontologia) e ANCP (Academia Nacional de Cuidados Paliativos) de alocação de recursos em esgotamento durante a pandemia por COVID-19. P. 4. Disponível em: https://www.amib.org.br/fileadmin/user_upload/amib/2020/abril/24/VJS01

${ }^{42}$ Keegan, MT e Soares, M. O que todo intensivista deveria saber sobre os sistemas de escore prognóstico e mortalidade ajustada ao risco. Rev Bras Ter Intensiva. 2016;28(3):264-269

${ }^{43}$ Versão um- Kretzer, L E et al. Protocolo AMIB de alocação de recursos em esgotamento durante a pandemia por COVID-19. P. 12. Disponível em:

https://www.amib.org.br/fileadmin/user upload/amib/2020/abril/24/Protocolo AMIB de alocacao de recursos em esgotamento durante a pandemia por COVID-19.pdf

${ }^{44}$ Dunham, AM E at al. A bioethical perspective for navigating moral dilemmas amidst the COVID-19 pandemic. The Journal of the American Academy of Orthopaedic Surgeons, 2020.

${ }^{45}$ BRASIL. Lei No 8142, de 28 de dezembro de 1990. Dispõe sobre a participação da comonidade na gestão do Sistema Único de Saúde (SUS) e sobre as transferências intergovernamentais de recursos financeiros na área da saúde e dá outras providências. 
${ }^{46}$ Casado $M$ E et al. RECOMENDACIONES PARA LA TOMA DE DECISIONES ÉTICAS SOBRE EL ACCESO DE PACIENTES A UNIDADES DE CUIDADOS ESPECIALES EN SITUACIONES DE PANDEMIA. 1st ed. Barcelona: Observatorio de Bioética y Derecho; 2020.

${ }^{47}$ Warrick, P. Questions of Life and Death : Who Lives? Decision by Committee. Los Angeles Times. 4 de aug. De 1991. https://www.latimes.com/archives/la-xpm-1991-08-04-vw-25story.html\#: :text=In\%20Seattle\%20in\%20the\%20summer,forerunner\%20of\%20modern\%20bioethics \%20committees.\&text=As\%20word\%20spread\%20that\%20Seattle,be\%20found\%20to\%20select\%20 patients.

48 Pincelli, R. [blog] 10 de jul. de 2011. https://www.blogs.unicamp.br/hypercubic/2011/07/o-conselhodos-deuses-de-seattle/

\section{${ }^{49}$ http://www.nephjc.com/news/godpanel}

50 Levine, C. [healthaffairs.org]. 30 de nov. de 2009.

https://www.healthaffairs.org/do/10.1377/hblog20091130.002998/full/

${ }^{51}$ Artigo escrito por Shana Alexander e publicado originalmente na revista LIFE, novembro de 1962.

${ }^{52}$ Dias, MC, Gonçalves L. Escolha sobre quem deve viver: bioética e COVID-19 no contexto brasileiro. Brasil de Fato. BH, 2020

${ }^{53}$ Silva et al.: Priority setting of ICU resources in an influenza pandemic: a qualitative study of the Canadian public's perspectives. BMC Public Health 2012 12:241., p.2.

${ }^{54}$ Silva et al.: Priority setting of ICU resources in an influenza pandemic: a qualitative study of the Canadian public's perspectives. BMC Public Health 2012 12:241.2012

${ }^{55}$ Rajan D E et al. Governance of the Covid-19 response: a call for more inclusive and transparent decision-making. BMJ Global Health 2020;5:e00265

${ }^{56}$ Dlas, M.C., G, L. Escolha sobre quem deve viver: bioética e COVID-19 no contexto brasileiro. Brasil de Fato. $\mathrm{BH}, 2020$

${ }^{57}$ Dias, MC, Gonçalves L. Escolha sobre quem deve viver: bioética e COVID-19 no contexto brasileiro. Brasil de Fato. BH, 2020

58 Versão um- Kretzer, L E et al. Protocolo AMIB de alocação de recursos em esgotamento durante a pandemia por COVID-19. Disponível em:

https://www.amib.org.br/fileadmin/user upload/amib/2020/abril/24/Protocolo AMIB de alocacao de recursos em esgotamento durante a pandemia por COVID-19.pdf

${ }^{59}$ Dias, MC, Gonçalves L. Escolha sobre quem deve viver: bioética e COVID-19 no contexto brasileiro. Brasil de Fato. BH, 2020

60 Núcleo de Operações e Inteligência em Saúde (NOIS) Nota Técnica 11 - 27/05/2020 Análise socioeconômica da taxa de letalidade da COVID-19 no Brasil. Acesso em: www.sites.google.com/view/nois-pucrio

${ }^{61}$ Brasil. Observatório Direitos Humanos e Crise Covid-19. Boletim informativo no 1, 2020. Disponível em:https://terradedireitos.org.br/uploads/arquivos/Boletim-1-Observatorio-DH-COVID19\%281\%29.pdf 
62 Dias, MC, Gonçalves L. Escolha sobre quem deve viver: bioética e COVID-19 no contexto brasileiro. Brasil de Fato. BH, 2020

${ }^{63}$ Dias, MC, Gonçalves L. Escolha sobre quem deve viver: bioética e COVID-19 no contexto brasileiro. Brasil de Fato. BH, 2020

${ }^{64}$ Bowen, W., G. O Curso do Rio. Rio de Janeiro: Garamond, 2004.

65 Dworkin, R. A Virtude Soberana: a Teoria e Prática da Igualdade. São Paulo. Editora WMF Martins Fontes. 2011.

${ }^{66}$ Satomi, E et al. Alocação Justa de Recursos de Saúde Escassos diante da Pandemia de COVID19: Considerações Éticas . Einstein (São Paulo). 2020; 18: eAE5775.

http://dx.doi.org/10.31744/einstein_journal/2020AE5775;

${ }^{67}$ Korupolu, R E et al. Reabilitation of Critically III COVID-19 Survivors. J Int Soc Phys Rehabil Med, 2020

${ }^{68}$ Sinuff T E et al. Values Ethics and Rationing in Critical Care Task Force. Rationing critical care beds: a systematic review. Crit Care Med. 2004;32(7):1588-97.

${ }^{69}$ Cesari, M. E Proietti, M. COVID-19 in Italy: Ageism and Decision Making. COVID-19 in Italy: Ageism and Decision Making in a Pandemic.J Am Med Dir Assoc ; 21(5): 576-577,2020.

70 AZEVEDO, M E et al. Por um Debate sobre as Diretrizes Éticas para Alocação de Tratamento em UTI durante a Pandemia. 17 de abril de 2020, disponível em https://estadodaarte.estadao.com.br/amplo-debate-criterios-uti-coronavirus/. Último acesso em $18 / 06 / 2020$. 